\title{
10 Jahre Organgruppe Mamma der Nordostdeutschen Gesellschaft für Gynäkologische Onkologie e.V. (NOGGO)
}

\author{
Jens-Uwe Blohmer ${ }^{\mathrm{a}} \quad$ Dirk Elling $^{\mathrm{b}} \quad$ Sherko Kümmel ${ }^{\mathrm{c}} \quad$ Jalid Sehouli ${ }^{\mathrm{d}}$ \\ Werner Lichtenegger $^{d}$ \\ ${ }^{a}$ Frauenheilkunde und Geburtshilfe, Brustzentrum-City, Berlin, \\ ${ }^{b}$ Frauenklinik, Sanaklinikum Berlin-Lichtenberg, \\ c Universitätsfrauenklinik, Essen, \\ ${ }^{\mathrm{d}}$ Frauenklinik, Universitätsklinikum Charité, Campus Virchow-Klinikum, Berlin, Germany
}

\section{Organisation der NOGGO e.V.}

Die NOGGO wurde am 27. März 1998 gegründet mit dem Ziel, zunächst in den neuen Bundesländern die Versorgung der Patientinnen mit einer gynäkologischonkologischen Erkrankung durch überregionale Fortbildung und die Durchführung von klinischen und experimentellen Studien zu verbessern. Im Laufe der Jahre wurden die Aktivitäten auf das gesamte Gebiet Deutschlands und weitere Länder Europas erweitert. Im Juni 2008 hatte die NOGGO mehr als 500 Mitglieder. Geleitet wird die NOGGO e.V. vom Vorstand (Vorstandsvorsitzender: Prof. Dr. med. Werner Lichtenegger), der vom Beirat unterstützt wird. Die Studienaktivitäten werden in den Arbeitsgruppen organisiert. Diese sind:

\section{AG Mammakarzinom}

(Leitung: Prof. Dr. med. Dirk Elling)

AG Ovarialkarzinom

(Leitung: Prof. Dr. med. Jalid Sehouli)

AG Zervixkarzinom

(Leitung: Prof. Dr. med. Werner Lichtenegger)

\author{
AG Endometriumkarzinom und uterine Sarkome \\ (Prof. Dr. med. Günter Köhler) \\ AG Supportive Therapien \\ (Dr. med. Hans-Joachim Hindenburg) \\ AG Translationale Forschung \\ (PD Dr. med. Carsten Denkert)
}

\section{Fortbildungen der NOGGO einschließlich der AG Mammakarzinom}

- Einmal pro Jahr «Post-ASCO» als Live-Konferenzschaltung aus Berlin (2008 zu 13 Kliniken in Deutschland, Österreich und der Schweiz).

- Einmal pro Jahr «Post-San Antonio Breast Cancer Symposium».

- Einmal pro Jahr: «Kontroversen in der Gynäkologischen Onkologie».

- Masterclass Ovarialkarzinom (Fortbildungsreihe mit Hospitationen).

- Einmal bis zweimal pro Jahr «Operatives Tutorial rekonstruktive und ästhetische Mammaoperationen» mit Vorträgen und gemeinsamen Operationen.

\begin{tabular}{ll}
\hline KARGER & ( ) 2008 S. Karger GmbH, Freiburg \\
Fax +497614520714 & Accessible online at: \\
$\begin{array}{l}\text { E-mail Information@Karger.de } \\
\text { www.karger.com }\end{array}$ & www.karger.com/brc \\
&
\end{tabular}




\section{Breast Care}

- 9.-11. Oktober 2008 «1. NOGGO-Hauptstadtkongress» mit dem Schwerpunkt Mammakarzinom. Dieser Kongress soll jedes zweite Jahr durchgeführt werden mit wechselnden Schwerpunkten der gynäkologischen Onkologie.

- Drei DEGUM-Mammasonographie-Kurse pro Jahr.

- Zertifiziertes Fortbildungscurriculum für Frauenärzte mit onkologischem Schwerpunkt (1 Mal pro Jahr).

- Fortbildungsreihen zur Palliativmedizin.

- Internationale Symposien auf internationalen Kongressen z.B. 17.-21.10.08 in Kos (8. International Conference of Anticancer Research).

\section{Fortbildungsmaterialien und Bücher, die die NOGGO für die Fortbildung bereit hält}

Folgende Literatur kann kostenlos von der NOGGO bezogen werden

- Update 2007/8. Chemotherapien in der Gynäkologischen Onkologie. Hrsg. Sehouli, J, Lichtenegger, W. Akademos Wissenschaftsverlag, 2008.

- Sehouli W, Lichtenegger W. Broschüre Ovarialkarzinom: Ratgeber für Patientinnen und deren Angehörigen. akademos Verlag, 1. Auflage 2002.

- Studienordner NOGGO

- Zusammenfassung: NOGGO-Intensivseminar: Internet-Nutzen für den gynäkologischen Onkologen?

- Zusammenfassung: NOGGO - State of the Art in der Gynäkologischen Onkologie. Standards und Ausblick für Klinik und Forschung.

- Zusammenfassung: NOGGO- State of the Art in der gynäkologischen Onkologie. Standards und Ausblick für Klinik und Forschung

- Zusammenfassung: NOGGO-Intensivworkshop: Grundprinzipien der Diagnostik und Behandlung des Mammakarzinoms - Standards und Ausblick.

- Sonstige: Verschiedene weitere Publikationen zur gynäkologischen Onkologie auf Anfrage.

\section{Wissenschaftliche Aktivitäten der AG Mammakarzinom der NOGGO}

Das Ziel jeder durchgeführten NOGGO-Studie ist, ihre Ergebnisse zu publizieren. Außerdem sollen, wenn möglich, Phase-III-Studien als Intergroup-Studien durchge- führt werden, um in kurzer Zeit die erforderliche Patientinnenzahl randomisieren zu können. Translationale Forschung soll in den Studienprotokollen integriert sein.

\section{Intergroup-Studien, an denen sich die AG Mamma-} karzinom der NOGGO beteiligt

GAIN: Multizentrische Phase-III-Studie zum Vergleich einer der beiden adjuvanten Chemotherapien ETC und EC-TX jeweils in Kombination mit Ibandronat oder Beobachtung bei Patientinnen mit nodalpositivem Mammakarzinom.

ICE: Klinische Studie zur Untersuchung der Wirkung von Ibandronat mit und ohne Capecitabin auf Brustkrebs bei älteren Patienten.

SUCCESS: Multizentrische, prospektiv randomisierte Phase-III-Studie zum Vergleich von FEC-DocChemotherapie versus FEC-DocG-Chemotherapie, sowie 2 oder 5 Jahre Zoledronattherapie in der adjuvanten Therapie von Patientinnen mit Brustkrebs.

GEPARQUINTO: Phase-III-Studienprogramm zur Kombination von Bevacizumab, Everolimus (RAD 001) oder Lapatinib mit einer neoadjuvanten Chemotherapie bei primärem Mammakarzinom.

ALLTO: Randomisierte, multizentrische, offene PhaseIII-Studie: Herceptin oder Lapatinib für 1 Jahr oder Herceptin und Lapatinib für $1 \mathrm{Jahr}$ oder Herceptin und 3 Monate, später Lapatinib dazu für 9 Monate.

NEO-ALLTO: Randomisierte, multizentrische, offene Phase-III-Studie zu neoadjuvant Lapatinib, Trastuzumab und deren Kombination plus Paclitaxel bei Frauen mit HER2/ErbB2-positivem, primärem Mammakarzinom.

Aktuell durchgeführte Studien der AG Mammakarzinom der NOGGO

LAPADO: Phase-I/II-Studie zur neoadjuvanten Chemotherapie mit nicht-pegyliertem liposomalem Doxorubicin, Paclitaxel und Lapatinib bei Patientinnen mit HER2-überexprimierenden, primären Mammakarzinomen. Studienleiter: D. Elling.

Phase-II-Studie beim metastasierten Mammakarzinom: Non-pegyliertes liposomales Doxorubicin und Paclitaxel. Studienleiter: S.-D. Costa.

Gen-Chip (MammaPrint)-gestützte Risikoanalyse von 400 Patientinnen mit einem frühen Mammakarzinom. 


\section{BreastCare}

\section{Publizierte Studien der AG Mammakarzinom der NOGGO}

Adjuvante Chemotherapie bei 1-3 positiven Lymphknoten, Phase-III-Studie mit translationaler Forschung 4 Zyklen Epirubicin 90 mit Paclitaxel 175 q2w gefolgt von 3 Zyklen CMF $(600,40,600)$ q2w vs. 4 Zyklen Epirubicin 90 mit Cyclophosphamid 600 q3w gefolgt von $3 \mathrm{Zy}$ klen CMF $(600,40,600)$ q3w bei Patientinnen mit 1-3 befallenen Lymphknoten (1-3 LK+).

Kümmel S, Eggemann H, Lüftner D, Gebauer N, Bühler H, Schaller G, Schmid P, Kreienberg R, Emons G, Kriner M, Elling D, Blohmer JU, Thomas A: Significant changes in circulating plasma levels of IGF1 and IGFBP3 after conventional or dose-intensified adjuvant treatment of breast cancer patients with one to three positive lymph nodes. Int J Biol Markers 2007; 22:186-93.

Kümmel S, Krocker J, Kohls A, Breitbach GP, Morack G, Budner M, Blohmer JU, Elling D: Randomised trial: survival benefit and safety of adjuvant dosedense chemotherapy for node-positive breast cancer. Br J Cancer 20068;94:1237-44.

Kümmel S, Eggemann H, Lüftner D, Thomas A, Jeschke S, Zerfel N, Heilmann V, Emons G, Zeiser T, Ulm K, Kobl M, Korlach S, Schmid P, Sehouli J, Elling D, Blohmer JU: Changes in the circulating plasma levels of VEGF and VEGF-D after adjuvant chemotherapy in patients with breast cancer and 1 to 3 positive lymph nodes. Anticancer Res 2006;26:1719-26.

Kümmel S, Krocker J, Kohls A, Breitbach GP, Morack G, Budner M, Blohmer JU, Lichtenegger W, Elling D: Dose-dense adjuvant chemotherapy for node-positive breast cancer in women 60 years and older: feasibility and tolerability in a subset of patients in a randomized trial. Crit Rev Oncol Hematol 2006;58:16675.

\section{Adjuvante Chemotherapie bei 4-9 positiven axillären} Lymphknoten, Phase-III-Studie

4 Zyklen Epirubicin 90 mit Paclitaxel 175 q2w gefolgt von 3 Zyklen CMF $(600,40,600)$ q2w vs. 4 Zyklen Epirubicin 90 mit Cyclophosphamid 600 q3w gefolgt von $3 \mathrm{Zy}$ klen CMF $(600,40,600)$ q3w bei Patientinnen mit 1-3 befallenen Lymphknoten (1-3 LK+).
Elling D, Krocker J, Kümmel S, Blohmer J, Lichtenegger W, Kohls A, Heinrich J, Quass J, Breitbach P, Köhler U. Dose-intensified adjuvant chemotherapy in high risk breast carcinoma with 4-9 positive lymph nodes. Zentralbl Gynakol.2000;122(4):207-16.

\section{Neoadjuvante Chemotherapie, Phase-II-Studien}

3 Zyklen Epirubicin 120 q2w gefolgt von 3 Zyklen Docetaxel 100 q2w bei T3 und T4d Mammakarzinomen.

Kümmel S, Thomas A, Paepke S, Schwarz M, Heinrich G, Wetzel A, Elling D, Kohls A, Lichtenegger W, Blohmer JU: Primary systemic chemotherapy with sequential, dose-dense epirubicin and docetaxel for inoperable, locally advanced inflammatory breast cancer: a phase II study. Acta Oncol 2005;44:248-54.

4 Zyklen Epirubicin 90 mit Ifosfamid 1,5 q2w neoadjuvant, gefolgt von 4 Zyklen Paclitaxel 175 adjuvant q 3w bei T3 und T4d Mammakarzinomen.

Blohmer JU, Paepke S, Kissner L, Elling D, Fleige B, Grineisen Y, Lichtenegger W: Dose-intensified, preoperative and adjuvant chemotherapy in patients with T3 and T4 breast cancer: toxicity, clinical and pathological remission. Zentralbl Gynakol 1999;121: $522-5$.

4 Zyklen Doxorubicin 60 mit Paclitaxel 200 q3w neoadjuvant bei T2 N0-1 Mammakarzinomen.

Schmid P, Krocker J, Morack G, Heilmann V, Blohmer JU, Michniewicz K, Köhler G, Schaller-Kranz T, Possinger K, Elling D: Primary chemotherapy with doxorubicin and paclitaxel in patients with early breast cancer: final results of a multicenter phase II study. J Cancer Res Clin Oncol 2004;130:657-63.

\section{Metastasiertes Mammakarzinom, Phase-III-Studie}

4 Zyklen Epirubicin 90 mit Cyclophosphamid 600 q3w vs. 4 Zyklen Epirubicin 75 mit Docetaxel 75 q3w beim viszeral metastasierten Mammakarzinom.

Blohmer JU, Lichtenegger W for the NOGGO: Multicenter randomized phase III study evaluating epirubicin/cyclophosphamide (ES) versus epirubicin/docetaxel (ED) for first line treatment of metastatic breast cancer (MBC): an interim analysis of safety and efficacy. Breast Cancer Res Treat 2002;76 (Suppl 1), \#333. 